\title{
Orally Administered Combination of Hyaluronic Acid, Chondroitin Sulfate, Curcumin, and Quercetin in the Prevention of Postcoital Recurrent Urinary Tract Infections: Analysis of 98 Women in Reproductive Age After 6 Months of Treatment
}

\author{
Michele Carlo Schiavi, MD, * Maria Grazia Porpora, MD, * Flaminia Vena, MD, * Giovanni Prata, MD, * \\ Valentina Sciuga, MD, * Ottavia D'Oria, MD, * Chiara Di Tucci, MD, * Delia Savone, MD, * \\ Natalia Aleksa, MD, * Andrea Giannini, MD, * Maria Paola Nusiner, MD, * Marzio Angelo Zullo, MD, $†$ \\ Ludovico Muzii, MD, * and Pierluigi Benedetti Panici, MD*
}

\begin{abstract}
Objective: The aim of this study is to assess the efficacy of orally administered combination of hyaluronic acid (HA), chondroitin sulfate (CS), curcumin, and quercetin for the prevention of postcoital recurrent urinary tract infection (UTI) in reproductive age women.

Methods: Ninety-eight consecutive patients in reproductive age affected by UTI were considered for the study. All 98 patients received a combination of HA, CS, curcumin, and quercetin two tablets per diem for the first month and one tablet every day for the next 5 months. We investigate recurrence of UTI with the Urinary Tract Infection Symptoms Assessment and the Pelvic Pain and Urinary Urgency Frequency. The quality of life and sexual function were valued using 36-Item Short Form Survey, Female Sexual Function Index, and the Female Sexual Distress Scale questionnaires. The same investigations were performed at the first visit and after 6 months of treatment.

Results: The symptoms associated with UTI significantly decreased after 6 months of treatment, in particular dysuria episodes diminished and number of voiding decreased $(P<0.0001)$. During the treatment period, only seven patients ( $7.1 \%$ ) experienced a UTI recurrence, confirmed by positive urine culture with bacteriuria of greater than $10^{5}$ colony forming units $/ \mathrm{mL}$. The Pelvic Pain and Urinary Urgency Frequency, Female Sexual Function Index, Female Sexual Distress Scale, and 36-Item Short Form Survey showed a statistically significant improvement after 6 months.

Conclusions: Oral administration of a combination of HA, CS, curcumin, and quercetin is a valid and well-tolerated nonantibiotic treatment for prevention of postcoital UTI in reproductive age women.
\end{abstract}

Key Words: recurrent urinary tract infections, hyaluronic acid, postcoital cystitis, quality of life, sexual function

(Female Pelvic Med Reconstr Surg 2018;00: 00-00)

$\mathrm{R}$ ecurrent uncomplicated urinary tract infection (rUTI) is a habitual presentation to gynecologists, urologists, and general practice doctors. A UTI indicates an infection of the urinary system producing an inflammatory response. A threshold of

From the *Department of Gynecological and Obstetric Sciences, and Urological Sciences, University of Rome "Sapienza," Umberto I Hospital; and †Department of Surgery-Week Surgery, Campus Biomedico, University of Rome, Rome, Italy.

Correspondence: Michele Carlo Schiavi, MD, Department of Gynecological and Obstetric Sciences, and Urological Sciences, University of Rome

"Sapienza," Umberto I Hospital, Viale del Policlinico 155, 00161 Roma.

E-mail: michelecarlo.schiavi@gmail.com.

The authors have declared they have no conflicts of interest.

This research did not receive any specific grant from funding agencies in the public, commercial, or not-for-profit sectors.

Copyright (C) 2018 Wolters Kluwer Health, Inc. All rights reserved.

DOI: $10.1097 /$ SPV.0000000000000560 three UTIs in 12 months or two episodes in the previous 6 months is used to define rUTI. ${ }^{1,2}$ The analysis literature data suggest that approximately one third of female population will have and require UTI treatment by age of 24 years and more than half will be affected in their lifetime. ${ }^{3}$

According to the literature, the principal risk factor for recurrence cystitis in a multivariate analysis was the frequency of sex act. ${ }^{4}$ Additional risk factors include spermicide use and/or new sex partner during the past year, a first UTI at or before 15 years of age, and a mother with a history of UTI. There is also an increased risk associated with urine-voiding disorders, such as those connected with prolapse, multiple sclerosis, bladder cancer, or bladder stones. A connection has been established with precoital and postcoital voiding, periodicity of urination, delayed voiding habits, douching, and body mass index. ${ }^{4}$

According to European Guidelines, management options are the following: continuous antibiotic prophylaxis, continuous prophylaxis with methenamine hippurate, cranberry products, passing urine after sex act, postcoital antibiotic prophylaxis and single-dose self-administered antibiotic, D-Mannose, probiotics, oral vaccine, ${ }^{5}$ and immunotherapy. ${ }^{6}$ Few data on long-term treatments currently support this contention.

Although antibiotic treatment is the most effective therapy, it selects for resistant uropathogens ${ }^{7}$ and commensal bacteria and adversely affects the gut, vaginal, and urinary microbiota, with different and relevant comorbidities. ${ }^{8,9}$ Furthermore, a long duration of cystitis symptoms could lead to a poor sexual function. ${ }^{8}$

Intravesical hyaluronic acid (HA) is an effective treatment with a remarkable security profile, ${ }^{10}$ but its invasive administration restricts its application in these women and there are few published randomized controlled trials. Therefore, the oral administration of HA could represent a new therapeutic option because of its ability to strengthen the urothelial coating. There is only one study that showed the efficacy of oral HA administration for the prevention of UTI, but only in climacteric women. ${ }^{11}$ The objective of the present study is to assess the efficacy of oral administration of a combination of HA, chondroitin sulfate (CS), curcumin, and quercetin for the prevention of postcoital rUTI in reproductive age women.

\section{MATERIALS AND METHODS}

From August 2016 to March 2017, 98 consecutive patients in reproductive age (mean [SD] age $=28.23$ [5.65]), affected by rUTI referred to the Department of Gynecological and Obstetrics Sciences, and Urological Sciences, University of Rome "Sapienza," were considered for the study. 
TABLE 1. Demographic and Clinical Characteristics of 98 Patients

\begin{tabular}{lr}
\hline Variables & \\
\hline Age, mean (SD), y & $28.23(5.65)$ \\
BMI, mean (SD) & $24.14(2.78)$ \\
Parity, median (range) & $1(1-2)$ \\
Previous pelvic surgery, n (\%) & $7(7.1)$ \\
Smoke, $\mathrm{n}(\%)$ & $21(21.4)$ \\
Previous UTI in the last 1 y, mean (SD) & $5.12(1.75)$ \\
New sex partners in the past year, median (range) & $2(2-3)$ \\
Intercourses in the past month, n (\%) & \\
$\quad>10$ times & $42(42.8)$ \\
$<10$ times & $56(57.2)$ \\
\hline
\end{tabular}

BMI, body mass index.

The present study is a retrospective analysis, and all data were retrospectively evaluated from a urogynecological internal database.

The inclusion criteria were as follows: women in reproductive age (18-45 years), a documented history of rUTI (3 UTIs in the previous 12 months or 2 episodes in the previous 6 months), cystitis after intercourse (postcoital cystitis), and a nonpositive urine culture at baseline. Recruited women were not in treatment with antibiotics for their rUTI since at the minimum 1 month.

The exclusion criteria were as follows: menopause, less than three uncomplicated UTIs in the previous 12 months or less than two in the previous 6 months, positive urine culture at the baseline, antibiotic therapy or other prevention method during the previous month, pelvic organ prolapse $\geq$ III stage (according to the pelvic organ prolapse quantification system system $),{ }^{12}$ significant $(>100 \mathrm{~mL})$ postvoid urine, stress or urgent urinary incontinence, neurologic bladder disease, interstitial cystitis/painful bladder syndrome, urinary tract stones, history or evidence of neoplastic disease, abnormalities of the urinary tract, renal insufficiency, diabetes mellitus, current corticosteroid use, and immunosuppressive disease.

The first visit consisted of a valuation of the following features: age, clinical history including frequency and timing of previous UTI, pathogens involved, the previous antibiotic regimens prescribed, clinical examination, urine culture, 3-day voiding diary, postvoid residual volume evaluation, baseline assessment of symptoms scores with Pelvic Pain and Urinary Urgency Frequency (PUF) patient symptom scale, ${ }^{13}$ and quality of life using the 36-Item Short Form Survey (SF-36). ${ }^{14}$ Urinary Tract Infection Symptoms Assessment (UTISA) validated questionnaire was administrated to enrolled patients ${ }^{15}$ : it is a 14 -item instrument asking about the severity of seven key UTI-related symptoms.

Sexual function was evaluated using Female Sexual Function Index (FSFI) ${ }^{16}$ and Female Sexual Distress Scale (FSDS). ${ }^{17}$

All 98 patients received an orally administered combination of HA, CS, curcumin, and quercetin two tablets daily for the first month and one tablet every days for the next 5 consecutive months. Each tablet contains precisely $200 \mathrm{mg}$ of curcumin complexed with soy phospholipids, $200 \mathrm{mg}$ of quercetin, $200 \mathrm{mg}$ of CS, and $20 \mathrm{mg}$ of $\mathrm{HA}$.

Patients were re-evaluated by the same investigations after 6 months of treatment. Adverse events were assessed and recorded.

The primary end point was the number of UTI recurrences during the 6 months of treatment. AUTI episode was defined by significant bacteriuria of greater than $10^{5}$ colony forming units $(\mathrm{CFU}) / \mathrm{mL}$ of midstream urine with or without clinical symptoms.

The secondary outcome was the variation of related urinary symptoms detected by the UTISA and PUF scores and finally the impact on quality of life and on sexual function evaluated with the SF-36, FSDS, and FSFI. The Patient Global Impression of Improvement after 6 months of treatment was calculated.

All values are presented as mean(SD). The number of UTIs was calculated using McNemar test. Paired, independent $t$ tests and the Pearson $x^{2}$ test were used to determine the significance of differences before and after treatment. A $P$ value of less than 0.05 was considered statistically significant. All analyses were conducted using the Statistical Package for the Social Sciences (22.0 for Mac; SPSS, Chicago, IL). Significance was set at a $P$ value of less than 0.05 .

\section{RESULTS}

Ninety-eight patients were recruited in the study. In the previous year, mean $(\mathrm{SD})$ number of $\mathrm{UTI}_{\mathrm{S}}$ was 5.12(1.75). Table 1 summarizes the other baseline epidemiologic and clinical characteristics of patients.

During the treatment period (6 months), seven patients (7.1\%) experienced a recurrence of their UTI, confirmed by positive urine culture with bacteriuria of greater than $10^{5} \mathrm{CFU} / \mathrm{mL}$. Of these patients, three $(3 \%)$ had more than ten sexual intercourses a month and four (4\%) had less than ten sexual intercourses a month without significant difference. No severe adverse effects were reported. Only three patients $(3 \%)$ experienced nausea during the treatment period. The mean number of positive urine culture decreased significantly after 6 months of treatment (3.84[2.34] vs 0.35 [0.52], $P<0.0001)$.

The mean number of symptoms associated with UTIs decreased significantly after treatment, in particular, dysuria episodes have decreased from 5.17(2.74) to 1.92(1.29), $P<0.0001$, and frequency diminished from 3.43(1.83) to 1.07(0.88), $P<0.0001$, after 6 months. The comparison of UTISA is shown in Table 2.

TABLE 2. Comparison of UTISA, Urine Culture, Quality of Life, and Sexual Function Questionnaires at Baseline (Last 1 Year) and at 6-Months FU After Treatment

\begin{tabular}{|c|c|c|c|}
\hline Variables & Baseline (Last 1 y) & 6-mo FU & $\boldsymbol{P}$ \\
\hline \multicolumn{4}{|c|}{ UTISA and urine culture } \\
\hline Dysuria & $5.17(2.74)$ & $1.92(1.29)$ & $<0.0001$ \\
\hline Increased frequency & $3.43(1.83)$ & $1.07(0.88)$ & $<0.0001$ \\
\hline Urgency & $4.87(1.53)$ & $1.34(1.56)$ & $<0.0001$ \\
\hline Tenesmus & $3.65(1.89)$ & $1.28(0.76)$ & $<0.0001$ \\
\hline Suprapubic pain & $4.76(1.35)$ & $1.72(0.77)$ & $<0.0001$ \\
\hline Backache & $2.11(0.75)$ & $1.55(1.12)$ & 0.002 \\
\hline Hematuria & $1.12(0.48)$ & $0.19(0.38)$ & $<0.0001$ \\
\hline Nocturia & $0.87(0.71)$ & $0.45(0.56)$ & $<0.0001$ \\
\hline Positive* urine culture & $3.84(2.34)$ & $0.35(0.52)$ & $<0.0001$ \\
\hline Antibiotic therapy & $4.65(2.43)$ & $0.89(0.65)$ & $<0.0001$ \\
\hline \multicolumn{4}{|c|}{ Quality of life questionnaires } \\
\hline PUF symptoms score & $14.81(4.87)$ & $7.54(4.12)$ & $<0.0001$ \\
\hline PUF bother score & $9.48(4.44)$ & $5.34(3.87)$ & $<0.0001$ \\
\hline PUF total score & $24.30(4.91)$ & $12.88(3.57)$ & $<0.0001$ \\
\hline SF-36 & $51.73(8.87)$ & $84.67(9.91)$ & $<0.0001$ \\
\hline FSFI & $20.73(2.87)$ & $28.46(1.97)$ & $<0.0001$ \\
\hline FSDS & $20.52(5.90)$ & $8.15(4.18)$ & $<0.0001$ \\
\hline \multicolumn{4}{|c|}{$\begin{array}{l}\text { Data are presented as mean (SD). } \\
\text { *Significant bacteriuria as }>10^{5} \mathrm{CFU} / \mathrm{mL} \text { of midstream urine. } \\
\mathrm{FU} \text {, follow-up. }\end{array}$} \\
\hline
\end{tabular}


TABLE 3. Patient Impression of Global Improvement After 6 Months of Treatment in 98 Patients

\begin{tabular}{lc}
\hline Variables & $\mathbf{n ~ ( \% )}$ \\
\hline 1. Very much better & $75(76.6)$ \\
2. Much better & $10(10.2)$ \\
3. A little better & $6(6.1)$ \\
4. No improvement & $7(7.1)$ \\
5. A little worse & 0 \\
6. Much worse & 0 \\
7. Very much worse & 0 \\
Success & $85(86.7)$ \\
\hline
\end{tabular}

The PUF total score and SF-36 have shown a statistically significant change respectively 24.30 (4.91) versus $12.88(3.57)$, $P<0.0001$, and 51.73(8.87) versus 84.67(9.91), $P<0.0001$, after 6 months of treatment as shown in Table 2.

No adverse effects and complications were reported during treatment. The overall satisfaction at Patient Impression of Global Improvement (very much better + much better) was reported in 85 patients $(86.8 \%)$ as shown in Table 3 .

\section{DISCUSSION}

The present study support the validity and security of oral association of HA, CS, curcumin, and quercetin to prevent recurrent and postcoital UTI in reproductive age women.

Urinary tract infection's recurrence has been associated to an alteration of glycosaminoglycans (GAGs) layer hatching the urothelial mucosa exposing the adhesion molecules and after permitting the incorporation of extracellular pathogen, particularly Escherichia Coli. Different studies sustained this hypothesis by providing the effectiveness of the intravesical instillation of GAGs/HA in preventing the recurrence of episodes. ${ }^{18,19}$ The possible gradual growing of antimicrobial resistance and the potential side effects of long-term antibiotic prophylaxis could explain GAG's role in protecting from bacterial rUTIs. Several studies have recently demonstrated the efficacy of intravesical hyaluronate treatment as an antibacterial biofilm agent. Although this procedure has been proven effective in reducing UTI, its invasiveness limits the compliance of treated patients. ${ }^{20-24}$ This preliminary study showed the efficacy and safety of oral HA in preventing lower UTI in reproductive age women affected by postcoital UTI. In the oral formulation, quercetin after being absorbed by the small intestine is changed into different metabolites, and the most active is quercetin-3-O- $\beta$-D-glucuronide. Its function is to decrease the expression of intercellular adhesion molecule 1 and other proinflammatory cytokines, obstructioning mast cell's degranulation, and lipid peroxidation. Another metabolite is tetrahydrocurcumin obtained from curcumin's hydrogenation. This element not only has antiinflammatory and antioxidant effect but also has an analgesic effect acting as transient receptor potential vanilloid l's antagonist. As shown previously, this oral compound is a valid treatment for recurrent UTIs. ${ }^{25-29}$ Oral HA has already been used in a low molecular weight pharmaceutical form for the prevention of stress urinary incontinence in women with atrophic vaginitis. ${ }^{30,31}$

Thanks to low molecular weight, we have a good effect on mucosal tissue (such as vaginal mucosa) and skin, with the guarantee of greater absorption and bioavailability. ${ }^{30}$ The action performed at the level of the bladder mucosa has been highlighted in our study where the important lowering in the number of postcoital UTI, the reduction of dysuria, urgency, antibiotic treatment, frequency, tenesmus, soprapubic pain, backache ematuria, nocturia, and positive urine culture was observed at 6 months' follow-up.
According to literature, the risk factors listed for rUTI are multiple. One of the most important is the frequency of sexual intercourse: there is almost a double risk for women who have more than ten sexual intercourses in the month before the recurrence of UTI. ${ }^{4}$ Contrariwise, in our study the risk of recurrence was not different among these groups of patients. Survey data suggest that the cystitis could be the result of a particularly painful sexual relationship for an unexperienced woman, poorly lubricated and with defensively contraction of the pelvic floor. ${ }^{8,32-35}$ There are data suggesting that routine postcoital antibiotic treatment is better than placebo and with the same efficacy as long-term antibiotic therapy ${ }^{36}$ in women with cystitis associated with sexual intercourse, but unfortunately, these substances trigger quick antibiotic resistance in uropathogens.

According to the available evidence-based medicine, current therapies for the prevention of rUTIs in young women include continuous or postcoital antibiotics prophylaxis, D-Mannose, probiotics, immunotherapy, and intravesical HA. ${ }^{11}$ Contrariwise, the latest literature data do not confirm the use of cranberry in preventing rUTIs ${ }^{9,36}$ (although it can be used as a equivalent therapy) and passing urine after sexual intercourse. As far as we know, our study is the first based drug prevention study in reproductive age women with oral administration of a combination of HA, CS, curcumin, and quercetin with a good safety profile, subjective improvements in patient well-being and sexual life (according to FSFI and FSDS), with most of patients reporting diagnostic improvement. This result could prove that the bettering of urinary symptoms and in particular the prevention of postcoital UTI decrease the risk of UTI during sexual intercourse and therefore women would be less afraid of having sex.

Although a positive effect was notified, our study has its limits, such as its retrospective nature, the small sample, and the deficiency of a placebo control group.

Further studies of oral administration of HA in prevention of rUTI on a larger number of cases, with a long-term follow-up and a placebo control group, should be performed to evaluate the duration of the therapeutic effects and to confirm our preliminary data. Larger researches should be performed regarding each component's metabolism and mechanism of action in oral administration.

In conclusion, this study suggests that oral assumption of a combination of HA, CS, curcumin, and quercetin is a valid, safe, and well-tolerated nonantibiotic treatment alternative for prevention in women with postcoital UTI.

\section{REFERENCES}

1. Epp A, Larochelle A, Lovatsis D, et al. Recurrent urinary tract infection. J Obstet Gynaecol Can 2010;32:1082-1101

2. Sen A. Recurrent cystitis in non-pregnant women. BMJ Clin Evid 2008; 7:801

3. Foxman B, Barlow R, D'Arcy H, et al. Urinary tract infection: self-reported incidence and associated costs. Ann Epidemiol 2000;10:509-515

4. Scholes D, Hooton TM, Roberts PL, et al. Risk factors for recurrent urinary tract infection in young women. J Infect Dis 2000;182(4):1177-1182.

5. Taha Neto KA, Nogueira Castilho L, Reis LO. Oral vaccine (OM-89) in the recurrent urinary tract infection prophylaxis: a realistic systematic review with meta-analysis. Actas Urol Esp 2016;40(4):203-208.

6. Ingersoll MA, Albert ML. From infection to immunotherapy: host immune responses to bacteria at the bladder mucosa. Mucosal Immunol 2013;6(6): 1041-1053.

7. Kamenski G, Wagner G, Zehetmayer S, et al. Antibacterial resistances in uncomplicated urinary tract infections in women: ECO $\cdot$ SENS II data from primary health care in Austria. BMC Infect Dis 2012;12:222.

8. Salonia A, Zanni G, Nappi RE, et al. Sexual dysfunction is common in women with lower urinary tract symptoms and urinary incontinence: results of a cross-sectional study. Eur Urol 2004;45(5):642-648. 
9. Jepson RG, Williams G, Craig JC. Cranberries for preventing urinary tract infections. Cochrane Database Syst Rev 2012;10:CD001321.

10. Lv YS, Zhou HL, Mao HP, et al. Intravesical hyaluronic acid and alkalinized lidocaine for the treatment of severe painful bladder syndrome/interstitial cystitis. Int Urogynecol J 2012;23:1715-1720.

11. Torella M, Del Deo F, Grimaldi A, et al. Efficacy of an orally administered combination of hyaluronic acid, chondroitin sulfate, curcumin and quercetin for the prevention of recurrent urinary tract infections in postmenopausal women. Eur J Obstet Gynecol Reprod Biol 2016;207: $125-128$.

12. Schiavi MC, Perniola G, Di Donato V, et al. Severe pelvic organ prolapse treated by vaginal native tissue repair: long-term analysis of outcomes in 146 patients. Arch Gynecol Obstet 2017;295(4):917-922.

13. Parsons CL, Dell J, Stanford EJ, et al. Increased prevalence of interstitial cystitis: previously unrecognized urologic and gynecologic cases identified using a new symptom questionnaire and intravesical potassium sensitivity. Urology 2002;60:573-578.

14. Burholt V, Nash P. Short form 36 (SF-36) health survey questionnaire: normative data for Wales. J Public Health (Oxf) 2011;33:587-603.

15. Clayson D, Wild D, Doll H, et al. Validation of a patient-administered questionnaire to measure the severity and bothersomeness of lower urinary tract symptoms in uncomplicated urinary tract infection (UTI) the UTI symptom assessment questionnaire. BJU Int 2005;96: 350-359.

16. Crisp CC, Fellner AN, Pauls RN, et al. Validation of the Female Sexual Function Index (FSFI) for web-based administration. Int Urogynecol J 2015;26(2):219-222.

17. Derogatis L, Clayton A, Lewis-D'Agostino D, et al. Validation of the female sexual distress scale-revised for assessing distress in women with hypoactive sexual desire disorder. $J$ Sex Med 2008;5(2): 357-64

18. Birder LA, de Groat WC. Mechanisms of disease: involvement of the urothelium in bladder dysfunction. Nat Clin Pract Urol 2007;4:46.

19. Parsons CL. The therapeutic role of sulfated polysaccharides in the urinary bladder. Urol Clin North Am 1994;21:93-100.

20. De Vita D, Antell H, Giordano S. Effectiveness of intravesical hyaluronic acid with or without chondroitin sulfate for recurrent bacterial cystitis in adult women: a meta-analysis. Int Urogynecol J 2013;24(4): $545-552$.

21. Lipovac M, Kurz C, Reithmayr F, et al. Prevention of recurrent bacterial urinary tract infections by intravesical instillation of hyaluronic acid. Int J Gynaecol Obstet 2007;96:192-195.

22. Constantinides C, Manousakas T, Nikolopoulos P, et al. Prevention of recurrent bacterial cystitis by intravesical administration of hyaluronic acid: a pilot study. BJU Int 2004;93(9):1262-1266.
23. Damiano R, Quarto G, Bava I, et al. Prevention of recurrent urinary tract infections by intravesical administration of hyaluronic acid and chondroitin sulphate: a placebo-controlled randomised trial. Eur Urol 2011;59:645-651.

24. Marcuzzo AV, Tofanelli M, Boscolo Nata F, et al. Hyaluronate effect on bacterial biofilm in ENT district infections: a review. APMIS 2017; $125: 763-772$.

25. Crespy V, Morand C, Manach C, et al. Part of quercetin absorbed in the small intestine is conjugated and further secreted in the intestinal lumen. Am J Physiol 1999;277(1 Pt 1):G120-G126.

26. Yan XM, Joo MJ, Lim JC, et al. The effect of quercetin-3-O- $\beta-D-$ glucuronopyranoside on indomethacin-induced gastric damage in rats via induction of mucus secretion and down-regulation of ICAM-1 expression. Arch Pharm Res 2011;34(9):1527-1534.

27. Pan MH, Huang TM, Lin JK. Biotransformation of curcumin through reduction and glucuronidation in mice. Drug Metab Dispos 1999;27(4): 486-494.

28. Osawa T, Sugiyama Y, Inayoshi M, et al. Antioxidative activity of tetrahydrocurcuminoids. Biosci Biotechnol Biochem 1995;59(9): 1609-1612.

29. Zhi L, Dong L, Kong D, et al. Curcumin acts via transient receptor potential vanilloid-1 receptors to inhibit gut nociception and reverses visceral hyperalgesia. Neurogastroenterol Motil 2013;25(6):e429-e440.

30. La Galia T, Micali A, Puzzolo D, et al. Oral low-molecular weight hyaluronic acid in the treatment of atrophic vaginitis. Int J Clin Med $2015 ; 617-624$.

31. Morelli M, Mocciaro R, Venturella R, et al. Hyaluronic acid-chondroitin sulfate: a potential factor to select pure stress urinary incontinence in patients with interstitial cystitisppainful bladder syndrome and mixed incontinence symptoms. Minerva Ginecol 2015;67(2):121-125.

32. Peters KM, Killinger KA, Carrico DJ, et al. Sexual function and sexual distress in women with interstitial cystitis: a case-control study. Urology 2007;70(3):543-547.

33. Donaldson RL, Meana M. Early dyspareunia experience in young women: confusion, consequences, and help-seeking barriers. J Sex Med 2011;8(3):814-823.

34. Coyne KS, Sexton CC, Thompson C, et al. The impact of OAB on sexua health in men and women: results from EpiLUTS. J Sex Med 2011;8(6): 1603-1615.

35. Sacco E, D'Addessi A, Racioppi M, et al. Bladder pain syndrome associated with highest impact on sexual function among women with lower urinary tract symptoms. Int J Gynaecol Obstet 2012;117(2): $168-172$.

36. Recurrent uncomplicated cystitis in women: allowing patients to self-initiate antibiotic therapy. Prescrire Int 2014;23(146):47-49. 